\title{
Deep Learning for Point Clouds Classification in the Ducal Palace at Urbino
}

\author{
Paolo Clini \\ Roberto Pierdicca \\ Ramona Quattrini \\ Emanuele Frontoni \\ Romina Nespeca
}

\section{Abstract}

Starting from a multi-scalar and multi-dimensional survey, most interdisciplinary researches, based on representation, are becoming a tool for dialogue between the new trends of Artificial Intelligence (Al) and the most compelling needs of our $\mathrm{CH}$. The approach here proposed stems from the desire to understand how much of the skills useful in architecture analysing and modelling can be made available to the "machine", with the goal to accelerate cognitive or management processes. Some $\mathrm{HBIM}$ models, as an existing digital heritage, were used to obtain the semantic intelligence. From this specialised intelligence comes a cyclical path which, through Al, transforms this knowledge into new forms of collective intelligence, at the service of the heritage. The paper presents a research that brings very promising results for the segmentation of point clouds and the facilitation of ScanToHBIM approaches, made possible by the large amount of data acquired on the Ducal Palace of Urbino.
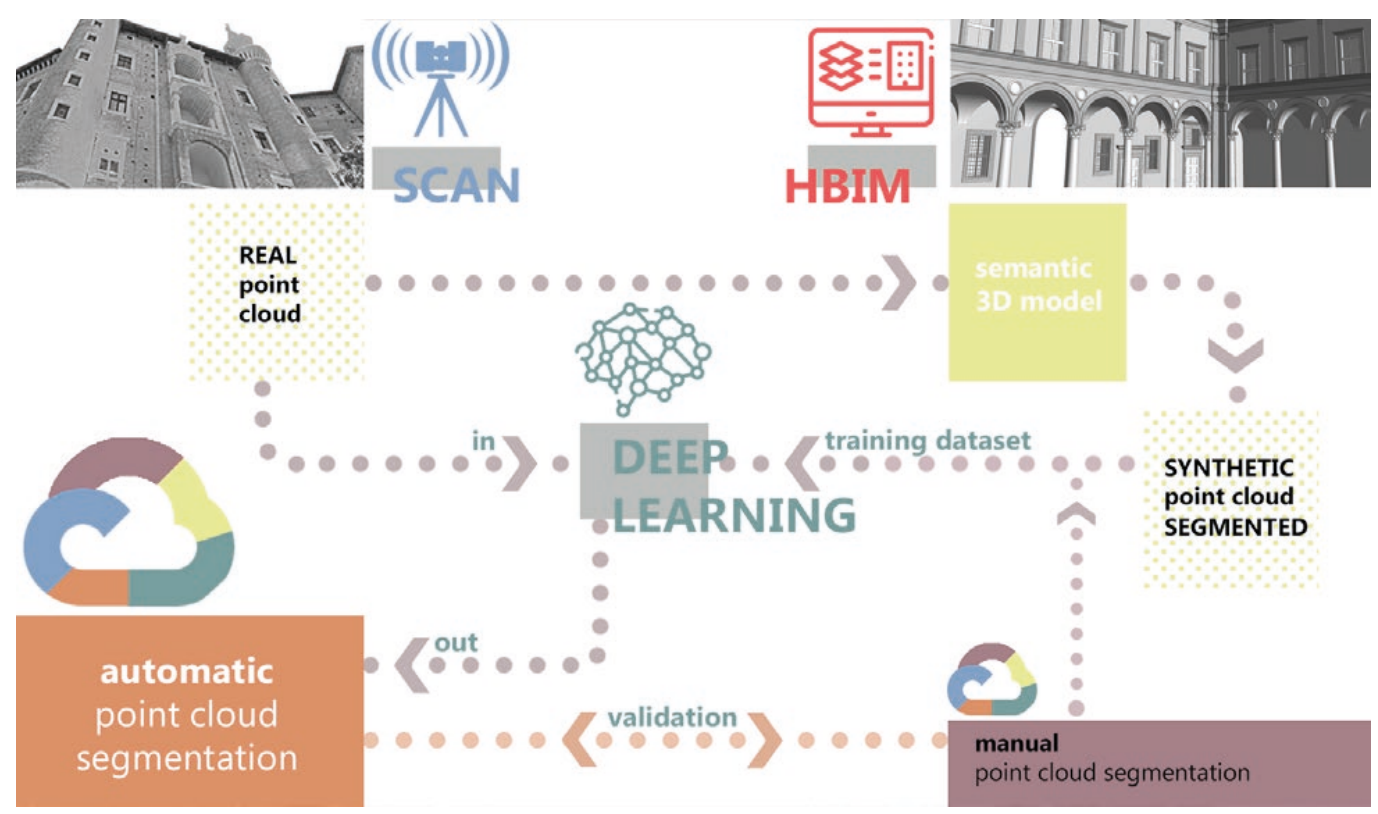


\section{Introduction}

The Digital Cultural Heritage $(\mathrm{DCH})$ stands today as a cornerstone in the processes of Built and Museum Heritage management and knowledge but also in its conservation. Starting from the current practices of multi-scalar and multi-dimensional survey, ranging from landscape and monumental heritage to artworks, the interdisciplinary research, based on representation, is becoming a tool for dialogue between the new trends of Artificial Intelligence (Al) and the most compelling needs of our heritage.

In the field of $\mathrm{CH}, \mathrm{Al}$ applications are dealing with both enabling new forms of digital data management, both generating digital assets from existing ones, with a surprising capacity of mimesis. However, a theoretical debate on the implications of $\mathrm{Al}$ in the context of Digital Humanities and Computational Modelling is still lacking.

The approach here proposed stems from the desire to understand how much of the skills useful in architecture analysing and modelling, inherent to the drawing discipline, can be made available to the 'machine', with the goal to accelerate cognitive or management processes with regard to the built heritage. Some HBIM models, considered as an existing digital heritage, were used to obtain the semantic intelligence. From this specialised intelligence comes a cyclical path which, through Al, transforms this knowledge into new forms of collective intelligence, at the service of the heritage.

The work is part of a broader strand of research in DCH by UNIVPM, now significantly developed in the strategic University project CIVITAS which addresses several challenges related to museums and the historical buildings.

\section{State of the Art}

The architectural heritage documentation is based on point clouds, with accurate discrete databases. However, in order to become effective, they need an informed and structured representation, mandatory based on semantic subdivision. For this reason, point clouds are often used, within BIM software, as a starting point for building parametric 3D, which incorporates semantic information and where architectural elements are identified and enriched with non-geometric information. This process, namely ScanToBIM, is costly and requires skilled operators.

The SACHER project obtained interesting BIM-independent results in the use of segmented 3D data [Bertacchi et al. 20I8, pp. 283-288], while the INCEPTION projects [ladanza et al. 2019, pp. 38I-388] and the BIM3DSG platform [Rechichi et al. 2016, pp. 703-7I0] achieved to interoperate and manage knowledge outside BIM platforms.

The need to automate, at least partially, the ScanToBIM process is largely agreed, certainly not in order to diminish the designer's knowledge but to make the whole process more agile focusing on new research challenges. Mainly the backbone is to make models more intelligent and aware of their nature.

An important step is the semantic segmentation, facilitating the identification of different types of architectural elements in the point clouds. It implies classifying each point to a particular type of object (e.g. wall, roof, column, vault, etc.).While Machine and Deep Learning techniques are spreading in every field, even at the basis of 'popular' applications with images labelling, point clouds make the task much more complex, especially when point clouds pertain to historical architecture. Machine Learning is also giving satisfactory results for datasets with different accuracies and hierarchical procedures [Teruggi et al. 2020, p. 2598]. To date deep learning is particularly challenging for classical architecture, due to the complexity of shapes and the limited repeatability of elements, making it difficult to define common patterns within the same class of elements [Pierdicca et al. 2020].

\section{The CIVITAS Project and Multi-Scalar Acquisition in the Ducal Palace at Urbino}

The paper presents a research that brings very promising results for the segmentation of point clouds and the facilitation of ScanToHBIM approaches, made possible by the large amount of data acquired on the Ducal Palace of Urbino and the collection of the National 
Gallery of the Marche region. The digitisation phase of the building and museum artefacts is a fundamental step of the CIVITAS project, as detailed in [Clini et al. 2020, pp. 194-228]. One of the challenges, in particular, is the optimisation of data management dealing with HBIM exploiting Linked Data, Semantic Web and Artificial Intelligence technologies, in order to perform new workflow starting from reality-based informed models.

The acquisitions implemented for the Ducal Palace of Urbino were based on a multi-scale approach, as highlighted in [Nespeca 2018, pp. I-14]: they tested various types of instrumental acquisition and defined the most appropriate levels of detail for the various forms of representation and features of the building. All the interior rooms and courtyards, but also the exterior of the building, were digitised with TLS, merged on a general 3D model acquired with Mobile Mapping and assisted by a campaign of spherical panoramas.

For many paintings and sculptures of the collection and for the Duke's Studiolo, dedicated photogrammetric acquisitions were deployed (fig. I), to complete the set of acquisitions. In this way, a comprehensive three-dimensional mapping was conducted, which forms the basis for any scientifically based action in the process of digital transformation of museums.

\section{Semantic Segmentation by Deep Learning Approach}

The point clouds, with the various gathered accuracies (fig. 2), constitute a high-quality morphometric model, but the nature and pertinence of the single points to the different components of the architecture is inferred by humans. Till recently the subdivision of point clouds was mostly based on algorithmic or manual approaches, now the possibilities granted by neural networks lead to exploit them to recognise points and assign them a semantics consistent with the rules of the architecture.

The identification of classes, which also has theoretical implications of great interest, has proved to be fundamental in the methodology. Following an analysis of coherence with the existing and consolidated thesauri and considering the historical period, its morphological-formal language and the features of the acquired data, a first level scale of the semantic hierarchy was defined (fig. 3).

The state of preservation of the building gives reason to expect very good correspondences with 'ideal' forms. The subsampling required to perform several tests and in several epochs, with an average computational capacity, also led to the choice of working on the most general level of the main architectural members.

A bottleneck in Deep Learning approaches applied to $\mathrm{CH}$ is the absence of sufficiently large annotated data sets that can allow the training of the networks. Thus, the approach took advantage of many previous models developed with semantic structuring features: from the Palladio Library villa models developed in 2012 to the most recent HBIM models (Ferretti Palace, Santa Maria of Portonovo and Ducal Palace). In addition, parametric models present
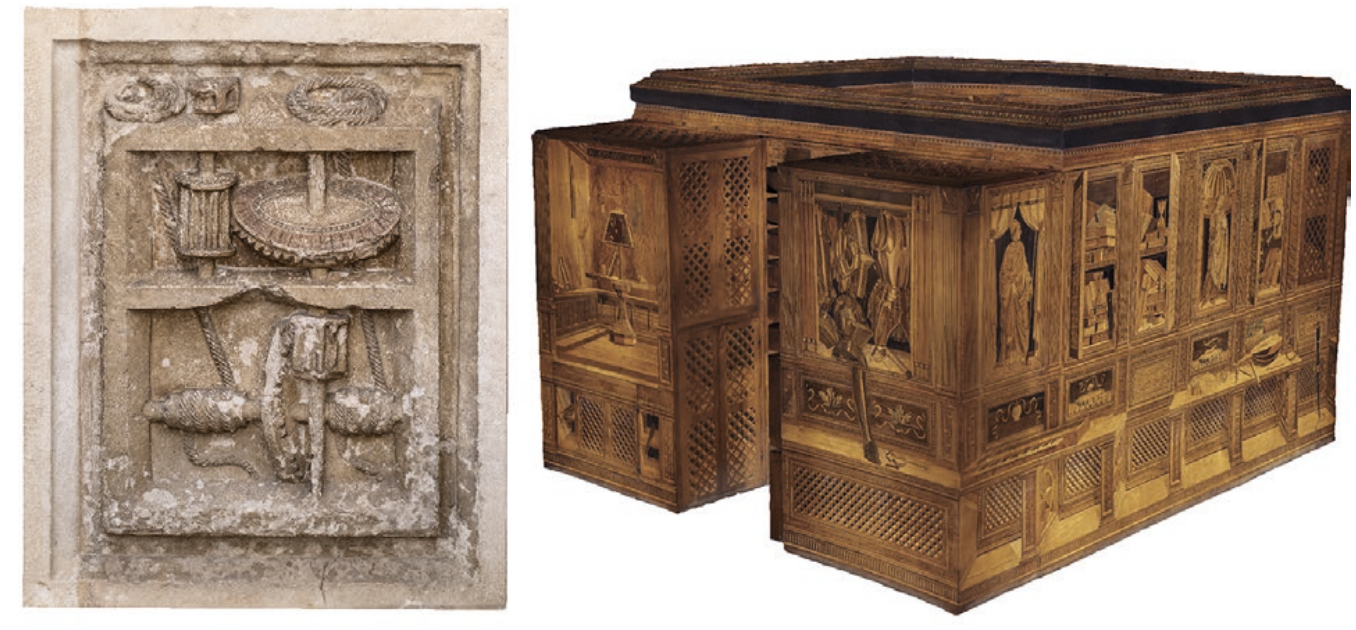
on the web were used and appropriately selected, as well as the HBIM core of the Honour Courtyard of Laurana. This allowed the generation of a sufficiently large data set of synthetic clouds to train the neural network. This phase therefore places drawing knowledge at the centre and the intelligence present in the models is regenerated and acquires new life and unexpected opportunities for value.

All the chosen models had the common characteristic of being organised according to a shapes grammar in which the constituent ontologies of the architectures had been analysed and studied. In the models, the classes were also consistent with those of the point clouds that were to be segmented, both in terms of formal qualities and hierarchical articulation. All models were stored in the various formats (.rvt, $3 \mathrm{dm}, \mathrm{kmz}$ ) and archived in a file format that incorporated the taxonomy by naming the layers. This enabled the export in .obj format and the subsequent creation of semantically structured synthetic point clouds.

At this point, the workflow for the Deep Learning approach foresees firstly the training of the neural network, i.e. its training, and then the experimentation on a never observed dataset. The DGCNN network was chosen, which is based on the EdgeConv operation, and also a refinement of it RaDGCNN, as better detailed in [Morbidoni et al. 2020a; 2020b].

Two experiments were performed on two different case studies: in the first one, we used the TLS point cloud of the courtyard of the Ducal Palace of Urbino to evaluate the trained models, trying to identify 8 different classes of architectural elements. For consistency, the synthetic point cloud derived from the HBIM model of the Ducal Palace of Urbino was removed from our training set.

In the second experiment we evaluated the models trained on the TLS point cloud of Ferretti Palace. In this case we removed the BIM model from the training set. Since two of the selected architectural element classes (column and pillar) are not present in the building, in this case we try to recognise the remaining 6 classes.

The results, reported here in qualitative form (fig. 4), allow us to conclude so far that the use of synthetic data can be effective in the automatic segmentation of TLS point clouds. Of course, this is only a first step and an encouraging scenario to be explored and analysed with other applications to support the ScanToBIM process of historic buildings.

\section{Conclusions}

In this article, a method, still in the process of being perfected, oriented towards the instruction of artificial intelligence for the segmentation of point clouds has been described: that is, an attempt has been made to improve the discernment capacities of neural networks, helping them with a form of collective intelligence built from the disciplinary foundations of design. These results in themselves constitute an interesting and novel approach, especially if we consider the potential for use and re-use of existing models, generated over the last 40 years or more, in terms of interoperability and sustainability of digitisation; but also in terms of formulating the axiom of digital heritage in itself, not as a mere copy.

Fig. 2. Point clouds of the Ducal Palace at Urbino.

Fig. 3. Identification of classes in the taxonomy
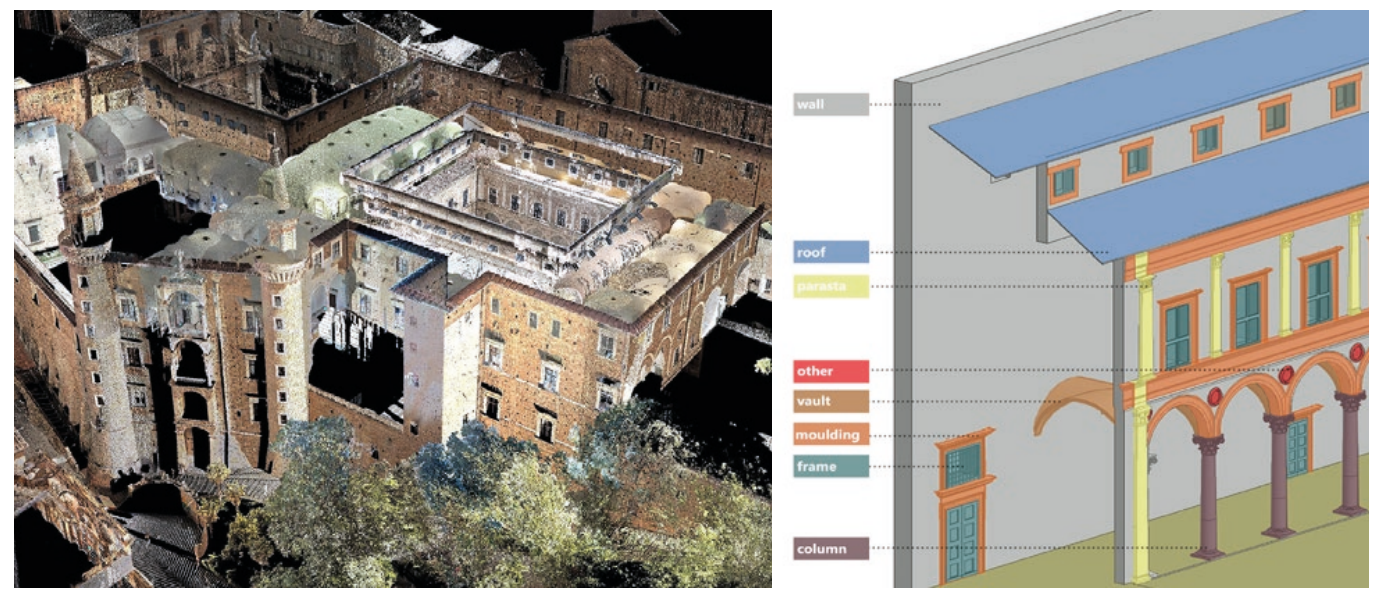
Fig. 4. Qualitative results about the segmentation of point clouds for the Ducal Palace.
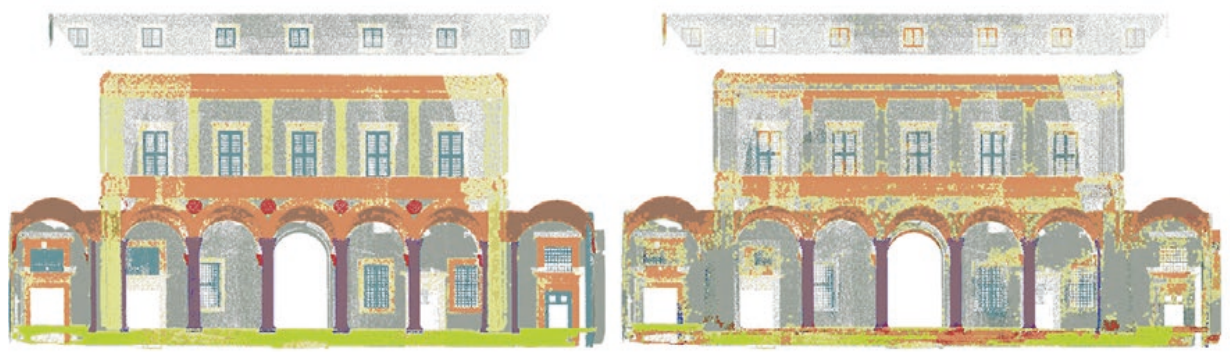

Another interesting food for thought comes from the essay [Clivaz 2020, pp. 67-73] in which Robert Wachal's 197I text is recalled. He raises what he sees as the main problem of the humanistic approach to computer science: to hope that the time will soon come when humanists will start asking new questions, also referring to artificial intelligence. Clivaz, too, emphasises that his 'personal vision' is an open appeal worthy of the attention of scholars today. So, also for us, $\mathrm{CH}$ experts, it is perhaps time to turn the telescope upside down: to start asking artificial intelligence new questions, the questions posed with increasing urgency by a fragile heritage.

\section{References}

Bertacchi Silvia, Al Jawarneh Isam Mashhour, Apollonio Fabrizio Ivan, Bertacchi Gianna, Cancilla Michele, Foschini Luca, Grana Costantino, Martuscelli Giuseppe, Montanari Rebecca (2018). SACHER Project. In Proceedings of the 4th EAI International Conference on Smart Objects and Technologies for Social Good - Goodtechs 'I 8. New York: ACM Press, pp. 283-288.

Clini Paolo, Quattrini Ramona, Bonvini Paolo, Nespeca Romina, Angeloni Renato, Mammoli Raissa, Dragoni Aldo Franco, Morbidoni Christian, Sernani Paolo, Mengoni Maura (2020). Digit (al) isation in Museums: Civitas Project-AR, VR, Multisensorial and Multiuser Experiences at the Urbino's Ducal Palace. In Virtual and Augmented Reality in Education, Art, and Museums. Hershey: IGI Global, pp. 194-228.

Clivaz Claire (2020). Digitized and Digitalized Humanities:Words and Identity. In Atti del IX Convegno Annuale AIUCD. La svolta inevitabile: sfide e prospettive per l'Informatica Umanistica. Milano: University del Sacre Cuore, pp. 67-73.

ladanza Ernesto, Maietti Federica, Ziri Anna Elisabetta, Di Giulio Roberto, Medici Marco, Ferrari Federico, Bonsma Paul,Turillazzi Beatrice (2019). Semantic Web Technologies Meet Bim for Accessing and Understanding Cultural Heritage. In International Archives of the Photogrammetry, Remote Sensing and Spatial Information Sciences, XLII-2N9 (2N9), pp. 38I-388.

Morbidoni Christian, Pierdicca Roberto, Paolanti Marina, Quattrini Ramona, Mammoli Raissa (2020a). Learning from synthetic point cloud data for historical buildings semantic segmentation. In Journal on Computing and Cultural Heritage. I 3 (4), pp. I - I6.

Morbidoni Christian, Pierdicca Roberto, Quattrini Ramona, Frontoni Emanuele (2020b). Graph CNN with radius distance for semantic segmentation of historical buildings TLS point clouds. In International Archives of the Photogrammetry, Remote Sensing and Spatial Information Sciences, XLIV-4/WI-2020, pp. 95-102.

Nespeca Romina (20I8). Towards a 3D digital model for management and fruition of Ducal Palace at Urbino. An integrated survey with mobile mapping. In SCIRES-IT - SClentific RESearch and Information Technology, 8 (2), pp. I- | 4.

Pierdicca Roberto, Paolanti Marina, Matrone Francesca, Martini Massimo, Morbidoni Christian, Malinverni Eva Savina, Frontoni Emanuele, Lingua Andrea Maria (2020). Point cloud semantic segmentation using a deep learning framework for cultural heritage. In Remote Sensing. 12 (6), p. I-23.

Rechichi Fabrizio, Mandelli Alessandro, Achille Cristiana, Fassi Francesco (2016). Sharing High-Resolution Models and Information on Web: The Web Module of Bim3DSG System. In International Archives of the Photogrammetry, Remote Sensing and Spatial Information Sciences. XLI-B5, pp. 703-7IO.

Teruggi Simone, Grilli Eleonora, Russo Michele, Fassi Francesco, Remondino Fabio (2020). A Hierarchical Machine Learning Approach for Multi-Level and Multi-Resolution 3D Point Cloud Classification. In Remote Sensing. I2 (I6), pp. -27.

\section{Authors}

Paolo Clini, Dept.of Civil Engineering, Construction and Architecture, Polytechnic University of Marche, p.clini@univpm.it Roberto Pierdicca, Dept. of Civil Engineering, Construction and Architecture, Polytechnic University of Marche, r.pierdicca@univpm.it Ramona Quattrini, Dept. of Civil Engineering, Construction and Architecture, Polytechnic University of Marche, r.quattrini@univpm.it Emanuele Frontoni, Dept.of Information Engineering, Polytechnic University of Marche, e.frontoni@univpm.it

Romina Nespeca, Dept. of Civil Engineering, Construction and Architecture, Polytechnic University of Marche, r.nespeca@univpm.it 
